\title{
Assessment of the Prognostic Role of Ki-67 and Its Optimal Cutoff Value in Early Breast Cancer: A Retrospective Analysis
}

\author{
Wael Makar, Shaimaa Lasheen
}

\begin{abstract}
Clinical Oncology Department, Kasr Al-Ainy Center of Clinical Oncology and Nuclear Medicine (NEMROCK), Kasr Al-Ainy School of Medicine, Cairo University, Cairo, Egypt
\end{abstract}

\begin{abstract}
Background: Breast cancer is a heterogenous group of diseases classified into the biological subtypes luminal A, luminal B, HER2-enriched and triple negative. These subtypes have different treatment response patterns and survival rates. Ki-67 is the most commonly used proliferative marker in breast cancer and is used for the distinction between luminal A and B subtypes.

Methods: A retrospective study that included patients with early breast cancer diagnosed between 2010 and 2016 and treated in a single cancer center.

Results: The medical records of 498 patients were retrospectively reviewed. The median age of patients was 51 years (range: 21 - 81) and the median value of Ki-67 level among them was 20\% (interquartile range: $10-30 \%$ ). Ki-67 was significantly higher in younger ( $<35$ years) and premenopausal patients ( $\mathrm{p}=0.0002$ and 0.0055 , respectively). Higher Ki-67 level associated significantly with higher $\mathrm{T}$ stage, estrogen and progesterone receptors-negativity, HER2-positivity and higher grade $(\mathrm{p}=0.0256,<0.0001,<0.0001,=0.0001$ and $=0.0031$; respectively). Univariate Cox regression analysis showed that the $\geq 14 \%$ and $\geq 20 \%$ cutoff values of Ki-67 level are associated with poorer diseasefree survival (DFS) $(\mathrm{HR}=1.989$ [95\%CI: 1.163-3.402, $\mathrm{p}=0.0121$ ] and $\mathrm{HR}=1.616$ [95\%CI: 1.001-2.61, p=0.0496], respectively). On stratifying patients according to the Ki-67 proliferation index into three strata, $<14 \%, \geq 14 \%--<20 \%$ and $\geq 20 \%$; DFS differed significantly between them $(\mathrm{p}=0.0394)$. The 5 -year DFS rate for the three strata was $82.2 \%$, $64.7 \%$ and $64.8 \%$; respectively.

Conclusion: Early breast cancer patients with lower Ki-67 levels have significantly better DFS. A Ki-67 cutoff value of $\geq 14 \%$ appears to correlate better with DFS than the newer cutoff value of $\geq 20 \%$.
\end{abstract}

Keywords: Biological subtypes, Cutoff value, Early breast cancer, Ki-67, Prognosis

Corresponding author: Wael Makar, MD; Clinical Oncology Department, Kasr Al-Ainy Center of Clinical Oncology and Nuclear Medicine (NEMROCK), Kasr Al-Ainy School of Medicine, Cairo University, Cairo, Egypt; Email: wael_makar@yahoo.com

Submitted: 2-April-2019, Revised: 14-Apr-2019, Accepted: 17-Apr-2019, Published online: 2-August-2020

(cc) BY

\section{Introduction}

Breast cancer is the most common malignancy in females affecting $38.8 \%$ of female patients diagnosed with cancer yearly, with an incidence rate of 35.8 for every 100,000 Egyptian females ${ }^{1}$. There are many factors with prognostic significance in breast cancer that have been investigated thoroughly in the last decades including patients' age, tumor size, nodal status, lymphovascular invasion, hormone receptor status and HER2 expression ${ }^{2}$. Breast cancer is considered a heterogenous disease. Based on hormonal receptors status and human epithelial growth factor receptor 2 (HER2) expression, it is classified into four biological subtypes: luminal $\mathrm{A}$ and $\mathrm{B}$, HER2-enriched and basal-like ${ }^{2}$. The luminal subtype (HR-positive) demonstrates an indolent disease with prolonged disease-free survival (DFS), low recurrence rates and sluggish response to chemotherapy. However, the emergence of Ki-67 proliferative index differentiated between two luminal subtypes; luminal A and luminal B breast cancer. These two subtypes showed different responses to chemotherapy and hormonal 
treatment as well as different patterns of recurrence and disease progression ${ }^{3}$. $\mathrm{Ki}-67$ is a non-histone nuclear protein expressed in all phases of the cell cycle except for the G0-phase i.e. a marker of cell proliferation ${ }^{3}$. In a large metaanalysis, Ki-67 expression in early breast cancer has been shown to be a significant prognostic indicator for DFS and overall survival (OS) when comparing node-negative / node-positive and untreated patients with Ki-67-positive expression regardless of the treatment options ${ }^{4}$. However, the Ki-67 proliferative index remains a controversial point in breast cancer prognosis of DFS and progression-free survival, especially with regards to its cutoff value of significance. Many studies have offered a cutoff value for significant Ki-67 level ranging from $14 \%$ to $20 \%{ }^{3,4}$.

Another challenge with the routine use of Ki-67 in clinical practice is the high inter- and intraobserver variability observed in testing. In spite of advanced immunohistochemical (IHC) methods, there is variation in human visual assessment of Ki67 level during the examination of specimens ${ }^{3,5}$.

The aim of this study was to evaluate the prognostic impact of Ki-67 on the outcome of early breast cancer as regards DFS and OS, and to determine which cutoff value $(14 \%$ vs. $20 \%)$ correlates better with outcome.

\section{Methods}

This retrospective study was conducted at a single Egyptian Cancer Center; Kasr Al-Ainy Center of Clinical Oncology and Nuclear Medicine (NEMROCK), Kasr Al-Ainy School of Medicine, Cairo University.

\section{Patients}

The medical records of patients treated at NEMROCK in the period from January 2010 to December 2016 were searched for breast cancer patients (identified through ICD-10 codes).

The criteria for inclusion in the study were: age $>18$, female gender, pathologically proven breast cancer, non-metastatic disease at presentation (stages I-III), available IHC results for estrogen receptors (ER), progesterone receptors (PR), HER2 and Ki-67, available baseline and follow-up clinical, radiologic and pathologic data and a minimum follow-up duration of 6 months. Patients with male gender, incomplete medical records or metastatic (stage IV) disease were excluded. Patients with no exact Ki-67 measurement and/or unknown HER2 status were also excluded.

For $\mathrm{T}$ staging, the clinical $\mathrm{T}$ for patients who received neoadjuvant treatment and the pathological $\mathrm{T}$ for other patients were taken into consideration.

HER2-negative breast cancer patients with weak ER/PR expression levels by IHC have survival outcomes similar to those with negative ER/PR expression ${ }^{6}$; consequently, they were considered as triple-negative subtype.

\section{Statistical Analysis}

Statistical analysis was performed using MedCalc version 19.4.0 (software for Windows MedCalc Software Ltd, Ostend, Belgium).

Categorical variables were described as number and percentage and abnormally distributed continuous variables as median and interquartile range (IQR). The Mann-Whitney U test was used to test the difference in Ki-67 expression value between two groups and the Kruskal-Wallis test for more than two groups.

The Kaplan-Meier method was used to estimate survival and to generate survival curves. Diseasefree survival was calculated from the date of diagnosis to the date of recurrence or death. Cox regression analysis or log-rank test was performed to test the association between variables and DFS. A $p$ value $<0.05$ was considered significant.

\section{Ethical Considerations}

The study protocol was reviewed and approved by the Ethics Committee of Kasr Al-Ainy Center of Clinical Oncology and Nuclear Medicine (NEMROCK), Kasr Al-Ainy School of Medicine, Cairo University.

\section{Results}

From 2,456 records of breast cancer patients in the specified time period, the data of 498 patients was complete and were eligible for analysis.

\section{Patients' and Disease Characteristics}

The median age of included patients was 51 years (range: $21-81$ ) and 241 (48.4\%) of them were premenopausal at diagnosis. Disease characteristics are mentioned in Table 1. One hundred and three (20.7\%) patients received neoadjuvant chemotherapy before surgery and 395 (79.3\%) received adjuvant therapy. The type of surgery was 
breast conservative surgery in 269 (54\%) patients and modified radical mastectomy in 227 (45.6\%). The type of surgery was missing in two patients.

Table 1: Disease characteristics

\begin{tabular}{|c|c|c|}
\hline Characteristic & No. & $\%$ \\
\hline \multicolumn{3}{|l|}{ T stage } \\
\hline $\mathrm{T} 1$ & 105 & 21.1 \\
\hline $\mathrm{T} 2$ & 274 & 55 \\
\hline T3 & 46 & 9.2 \\
\hline $\mathrm{T} 4$ & 30 & 6 \\
\hline Missing & 43 & 8.6 \\
\hline \multicolumn{3}{|l|}{ N stage } \\
\hline N0 & 223 & 44.8 \\
\hline N1 & 128 & 25.7 \\
\hline $\mathrm{N} 2$ & 76 & 15.3 \\
\hline N3 & 56 & 11.2 \\
\hline Missing & 15 & 3 \\
\hline \multicolumn{3}{|l|}{ Grade } \\
\hline 1 & 3 & 0.6 \\
\hline 2 & 333 & 66.9 \\
\hline 3 & 42 & 8.4 \\
\hline Missing & 120 & 24.1 \\
\hline \multicolumn{3}{|c|}{ Lymphovascular invasion } \\
\hline No & 229 & 46 \\
\hline Yes & 135 & 27.1 \\
\hline Missing & 134 & 26.9 \\
\hline \multicolumn{3}{|l|}{ Estrogen receptor status } \\
\hline Negative & 126 & 25.3 \\
\hline Weakly positive & 34 & 6.8 \\
\hline Moderately positive & 73 & 14.7 \\
\hline Strongly positive & 265 & 53.2 \\
\hline \multicolumn{3}{|c|}{ Progesterone receptor status } \\
\hline Negative & 167 & 33.5 \\
\hline Weakly positive & 50 & 10 \\
\hline Moderately positive & 97 & 19.5 \\
\hline Strongly positive & 184 & 36.9 \\
\hline \multicolumn{3}{|l|}{ HER2 } \\
\hline Negative & 385 & 77.3 \\
\hline Positive & 113 & 22.7 \\
\hline \multicolumn{3}{|l|}{ Ki-67 } \\
\hline$<14$ & 191 & 38.4 \\
\hline$\geq 14-<20$ & 55 & 11 \\
\hline$\geq 20$ & 252 & 50.6 \\
\hline
\end{tabular}

In our sample, Ki-67 measured in surgical specimens had a median of $20 \%$ (IQR: $10-30 \%)$. The distribution of Ki-67 measurements is shown in Figure 1.

\section{Correlation Between Ki-67 and Clinico- Pathological Variables}

The relation between Ki-67 and the other variables is shown in Table 2. Ki-67 level was significantly higher in premenopausal and younger patients. Higher T-stage, estrogen and progesterone-negativity, HER2-positivity and higher grade were also associated with significantly higher Ki-67.

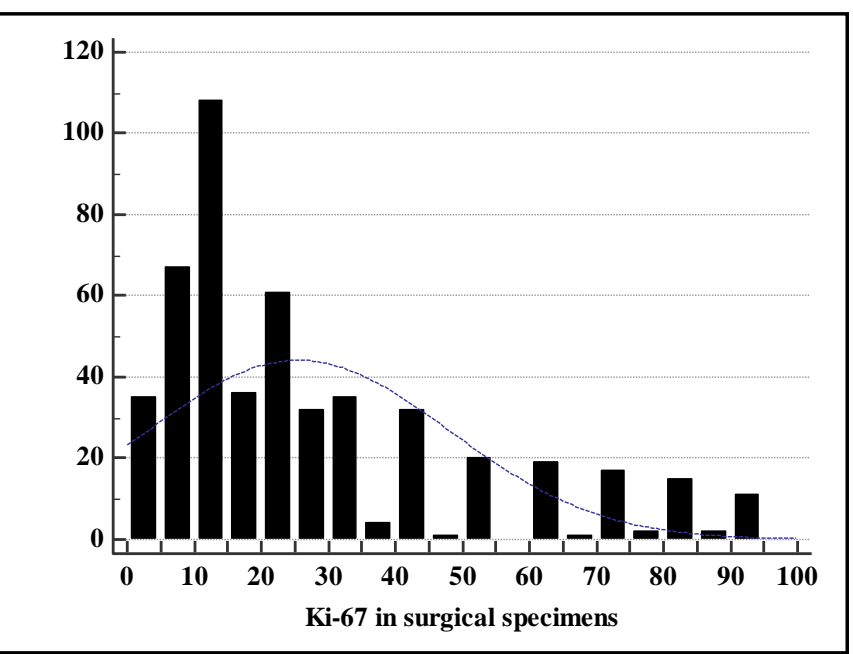

Figure 1: Histogram showing the distribution of Ki-67 expression in 498 early breast cancer patients

\section{Survival Analysis}

After a median follow up time of 24.6 months (95\%CI: 19.93-67.57), 71 (14.3\%) had relapsed disease and only 6 (1.2\%) patients died.

The estimated mean OS was 66.3 months (95\%CI: 65.24-67.36) and the estimated median was not reached. No further OS analysis was performed because of the very few number of events (deaths). The estimated mean DFS was 56 months $(95 \% \mathrm{CI}$ : 53.58-58.41) and the median was 67.57 months (95\%CI: 67.57-67.57).

Univariate DFS analysis using Cox proportional hazards model is shown in Table 3. Being young (< 35 years) and premenopausal were associated with significantly worse DFS. This was also true for nodal positivity, higher grade, lymphovascular invasion, and ER and PR negativity. Although higher T stage and HER2 positivity were associated with worse DFS, the association was not statistically significant.

Cox proportion hazards model was used after stratification of patients according to the Ki-67 level using the cutoff value of $14 \%$. Using the $14 \%$ cutoff value for Ki-67 ( $\geq 14 \%$ vs. $<14 \%)$, there was a significant correlation to DFS (HR=1.989 [95\%CI: 1.163-3.402]; $\mathrm{p}=0.0121$ ) (Figure 2). The 5-year DFS rate was $65 \%$ for Ki-67 $\geq 14 \%$ and $82.2 \%$ for Ki- $67<$ $14 \%$.

When $20 \%$ was used as a cutoff value for Ki-67, there was also a significant correlation to DFS 
(HR=1.616 [95\%CI: 1.001-2.61], p=0.0496) (Figure 3). The 5-year DFS rate was $64.8 \%$ for Ki-67 $\geq 20 \%$ and $78.3 \%$ for Ki-67 $<20 \%$. On stratifying the Ki-67 proliferation index into three strata i.e. $<14 \%$, $\geq$ $14 \%--<20 \%$ and $\geq 20 \%$, DFS showed a statistically significant difference between the 3 strata $(p=0.0394)$ (Figure 4).

Table 2: Correlation between Ki-67 and clinicopathological variables

\begin{tabular}{|c|c|c|}
\hline Variable & $\begin{array}{l}\text { Ki-67 } \\
\text { Median (IQR) }\end{array}$ & $\begin{array}{l}\mathrm{P} \text { - } \\
\text { value }\end{array}$ \\
\hline \multicolumn{3}{|l|}{ Age } \\
\hline$<35$ & $30(15-60)$ & \multirow[t]{2}{*}{0.0002} \\
\hline$\geq 35$ & $18(10-30)$ & \\
\hline \multicolumn{3}{|l|}{ Menopause } \\
\hline Premenopausal & $20(10-40)$ & \multirow[t]{2}{*}{0.0055} \\
\hline Postmenopausal & $15(10-30)$ & \\
\hline \multicolumn{3}{|l|}{ T stage } \\
\hline $1-2$ & $18(10-30)$ & \multirow[t]{2}{*}{0.0256} \\
\hline $3-4$ & $20(11-40)$ & \\
\hline \multicolumn{3}{|l|}{ N stage } \\
\hline 0 & $17(9-38.8)$ & \multirow[t]{2}{*}{0.4367} \\
\hline $1-3$ & $20(10-30)$ & \\
\hline \multicolumn{3}{|l|}{ Estrogen receptor } \\
\hline Negative & $27.5(14-60)$ & \multirow[t]{4}{*}{$<0.0001$} \\
\hline Weakly positive & $22.5(10-60)$ & \\
\hline Moderately positive & $18(10-30)$ & \\
\hline Strongly positive & $15(10-25)$ & \\
\hline \multicolumn{3}{|l|}{$\begin{array}{l}\text { Progesterone } \\
\text { receptor }\end{array}$} \\
\hline Negative & $25(14-50)$ & \multirow[t]{4}{*}{$<0.0001$} \\
\hline Weakly positive & $16.5(10-30)$ & \\
\hline Moderately positive & $15(10-30)$ & \\
\hline Strongly positive & $12(9.5-25)$ & \\
\hline \multicolumn{3}{|l|}{ HER2 } \\
\hline Negative & $15(10-30)$ & \multirow[t]{2}{*}{0.0001} \\
\hline Positive & $20(14-40)$ & \\
\hline \multicolumn{3}{|l|}{ Grade } \\
\hline $1-2$ & $17(10-30)$ & \multirow[t]{2}{*}{0.0031} \\
\hline 3 & $25(15-60)$ & \\
\hline
\end{tabular}

IQR: Interquartile range

The 5-year DFS rate for the three categories was $82.2 \%, 64.7 \%$ and $64.8 \%$; respectively. As shown in Figure 4, the DFS curve of the Ki-67 $\geq 14 \%--<20 \%$ group was overlapping with the $\geq 20 \%$ group curve but not the $<14 \%$ group curve.

In multivariate Cox regression analysis that included significant variables in Table 3, Ki-67 cutoff value of $14 \%$ did not associate significantly with DFS (HR=1.354 [95\%CI: 0.663-2.762], $\mathrm{p}=0.405$ ). The same was true for the cutoff value of $20 \%$ (HR=1.376 [95\%CI: 0.723-2.619], $\mathrm{p}=0.33$ ). In the multivariate analysis, age, nodal involvement and ER and PR status maintained significance.

Table 3: Univariate analysis for disease-free survival

\begin{tabular}{|c|c|c|}
\hline Variable & HR (95\%CI) & P-value \\
\hline \multicolumn{3}{|l|}{ Age } \\
\hline$<35$ & Ref. & \multirow[t]{2}{*}{$<0.0001$} \\
\hline$\geq 35$ & $0.282(0.159-0.499)$ & \\
\hline \multicolumn{3}{|l|}{ Menopausal status } \\
\hline Postmenopausal & Ref. & \multirow[t]{2}{*}{0.0092} \\
\hline Premenopausal & $1.917(1.175-3.126)$ & \\
\hline \multicolumn{3}{|l|}{ T stage } \\
\hline $1-2$ & Ref. & \multirow[t]{2}{*}{0.6917} \\
\hline $3-4$ & $1.155(0.566-2.359)$ & \\
\hline \multicolumn{3}{|l|}{ N stage } \\
\hline 0 & Ref. & \multirow[t]{2}{*}{$<0.0001$} \\
\hline $1-3$ & $3.479(1.925-6.288)$ & \\
\hline \multicolumn{3}{|l|}{ Grade } \\
\hline $1-2$ & Ref. & \multirow[t]{2}{*}{0.0313} \\
\hline 3 & $2.211(1.074-4.552)$ & \\
\hline \multicolumn{3}{|l|}{$\begin{array}{l}\text { Lymphovascular } \\
\text { invasion }\end{array}$} \\
\hline No & Ref. & \multirow[t]{2}{*}{0.0087} \\
\hline Yes & $2.006(1.193-3.374)$ & \\
\hline \multicolumn{3}{|l|}{ Estrogen receptor } \\
\hline Negative & Ref. & \multirow[t]{2}{*}{0.0038} \\
\hline Positive & $0.49(0.302-0.795)$ & \\
\hline \multicolumn{3}{|l|}{$\begin{array}{l}\text { Progesterone } \\
\text { receptor }\end{array}$} \\
\hline Negative & Ref. & \multirow[t]{2}{*}{0.0456} \\
\hline Positive & $0.619(0.387-0.991)$ & \\
\hline \multicolumn{3}{|l|}{ HER2 amplification } \\
\hline No & Ref. & \multirow[t]{2}{*}{0.2766} \\
\hline Yes & $1.334(0.794-2.243)$ & \\
\hline
\end{tabular}

HR: Hazard ration; CI: Confidence interval

\section{Discussion}

To our knowledge, this is the biggest study of the prognostic impact of Ki-67 in breast cancer performed in Egypt. Multiple prognostic and predictive markers of breast cancer outcome have been identified over the last few decades. Among these, Ki-67 arises as an important prognostic factor currently integrated into routine clinical practice.

Despite of that, a lot of controversy is raised around the routine use of Ki-67 especially with the lack of consistent evidence of its predictive value regarding the use of adjuvant systemic treatment.

The technical challenges and high discordance rate of Ki-67 reporting among pathologists (interand intra - observer variation) poses another 


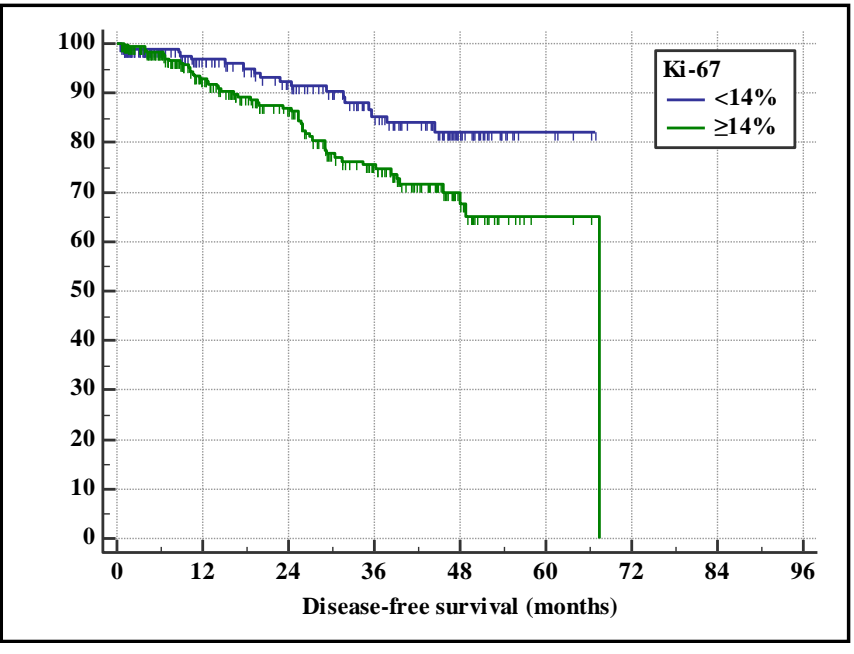

Figure 2: Kaplan-Meier disease-free survival estimates according to Ki-67 cutoff value of $14 \%$

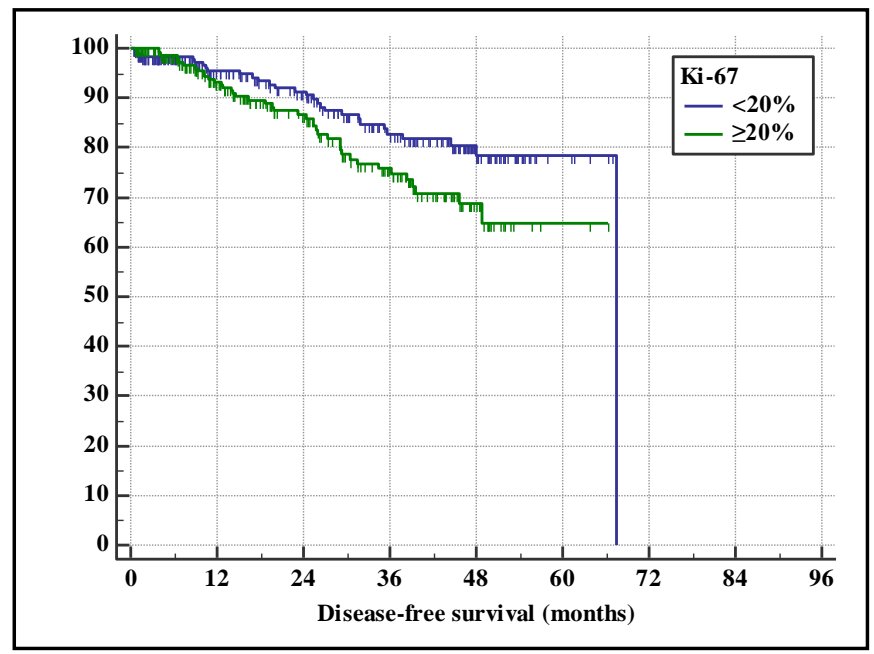

Figure 3: Kaplan-Meier disease-free survival estimates according to Ki-67 cutoff value of $20 \%$

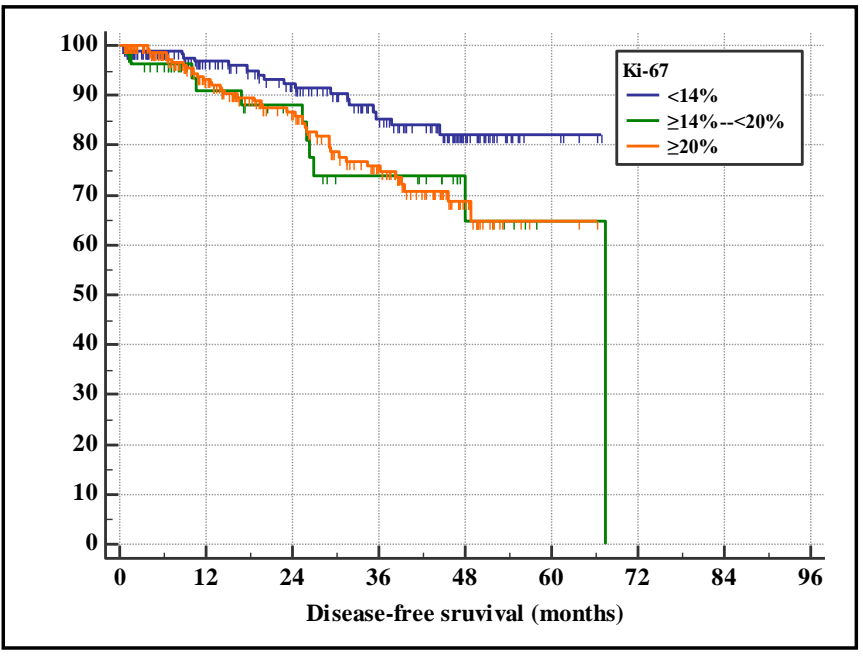

Figure 4: Kaplan-Meier disease-free survival estimates according to Ki-67 stratified into 3 categories challenge for the routine use of $\mathrm{Ki}-67$ in clinical practice.

Furthermore, the debate regarding the optimum Ki-67 cutoff value of clinical significance is another challenge facing the use of Ki-67 in clinical practice. The use of Ki-67 was first suggested in the St. Gallen consensus meeting in 2009 to identify highly proliferating tumors within Luminal breast cancers. In 2011, the consensus meeting suggested a cutoff value of $14 \%$ based on the data reported by Cheang et al 2; however, in 2013 the suggested cutoff value was changed to $20 \%$.

In the current study, we aimed to evaluate the prognostic impact of $\mathrm{Ki}-67$ on breast cancer outcome, as well as to correlate different cutoff levels of Ki-67 to survival to elucidate the optimum cutoff level of clinical significance at our institute, especially with the limited use of gene expression profiling due to financial restrictions.

In the current study, Ki-67 did correlate neither to tumor size nor to nodal status. This is consistent with the data reported by Inwald et al revealing no correlation between Ki-67 and T-stage, while it did correlate to N-stage ${ }^{7}$. However, many studies demonstrated the reverse; with a positive correlation between Ki-67 and both ${ }^{4}$. Kremani et al found a marginal significant relationship between lymph node status and Ki-67 expression ${ }^{8}$.

On the other hand, in our study Ki-67 correlated significantly to ER, PR and HER2 status. Mirmalek et al stated in their study that age, ER and PR status correlated negatively with Ki-67, while HER2 correlated positively ${ }^{9}$. Estrogen receptor and PR had an inverse relationship with Ki-67 in the study of Sheikhpour et al ${ }^{10}$. Our study results showed a significant correlation to younger age ( $<35$ years), and accordingly to being premenopausal, similar to other studies done across Egypt ${ }^{1}$.

When we used a cutoff value of $<14 \%$ to evaluate the impact of Ki-67 on breast cancer outcome, a positive correlation to DFS was statistically significant. This is consistent with the results of Hugh et al who studied 1,350 patients using the same cutoff value of $14 \%$ and found a significant improvement in 3-year DFS rate in patients with Ki67 less than $14 \%{ }^{11}$. Cheang et al ${ }^{2}$, Chung et al ${ }^{12}$, Thangarajah et al ${ }^{13}$ and Yang et al ${ }^{14}$ also reported similar results with the use of the same cutoff value of $14 \%$.

When we performed the analysis using a Ki-67 cutoff value of $20 \%$, similar results were obtained with significant correlation to DFS. This concords with the data reported by Bustreo et al on 1,577 
patients where patients with Ki-67 > 20\% had a poorer prognosis ${ }^{3}$. Accordingly, we stratified our patient cohort into three groups according to Ki-67 into those with an index of $<14 \%, \geq 14 \%--<20 \%$ and $\geq 20 \%$. This resulted in a significant difference in DFS was between the three groups with patients with Ki-67 $\leq 14 \%$ showing the best outcome. This is consistent with the suggestion of Denkert et al ${ }^{15}$ that Ki-67 can be considered as a continuous marker because of its prognostic significance using different cutoff values. The 5-year DFS rates of patients in the Ki-67 $\geq 14 \%--<20 \%$ and $\geq 20 \%$ groups were similar and significantly lower than that of the $<14 \%$ group.

In our study, we faced some limitations such as incomplete medical records of patients who had not completed their treatment at our center and the incomplete panel of IHC. In addition, in many patients, high and low readings were interpreted according to the $14 \%$ cutoff value that was previously recommended by St. Gallen consensus in 2011. Furthermore, our study was a retrospective one from a single cancer center with limited followup duration.

\section{Conclusion}

The present study further confirms the prognostic impact of Ki-67 on early breast cancer outcome, and that a Ki-67 cutoff value of $14 \%$ correlates better with DFS.

\section{Acknowledgment \\ None.}

\section{Author's contribution}

Conception or design: WM and SL; Acquisition, analysis or interpretation of data: SL; Drafting the manuscript or revising it: WM and SL; Final approval of the manuscript version to be published: WM and SL; Agreement to be accountable for all aspects of the work: WM and SL.

\section{Conflict of interest}

The authors declare that they have no conflict of interest to disclose.

\section{Data a vailability}

Deidentified individual participant data used to produce the results of this study are available from the corresponding author (WM) upon request.

\section{Funding}

The authors did not receive funding for this study.

\section{Study registration}

None.

\section{References}

1. Ibrahim AS, Khaled HM, Mikhail NN, Baraka H, Kamel H. Cancer incidence in Egypt: Results of the National population-based cancer registry program. J Cancer Epidemiol. 2014; 2014:437971.

2. Cheang MC, Chia SK, Voduc D, et al. Ki-67 index, HER2 status, and prognosis of patients with luminal B breast cancer. J Natl Cancer Inst. 2009; 101(10): 736-750.

3. Bustreo S, Osella-Abate S, Cassoni P, et al. Optimal Ki67 cutoff for luminal breast cancer prognostic evaluation: a large case series study with a long-term follow-up. Breast Cancer Res Treat. 2016; 157(2): 363371

4. Acs B, Zambo V, Vizkeleti L, et al. Ki-67 as a controversial predictive and prognostic marker in breast cancer patients treated with neoadjuvant chemotherapy. Diagn Pathol. 2017; 12(1): 20.

5. de Azambuja E, Cardoso F, de Castro G Jr, et al. Ki-67 as prognostic marker in early breast cancer: a metaanalysis of published studies involving 12,155 patients. Br J Cancer. 2007; 96(10): 1504-1513.

6. Liao GS, Dai MS, Hsu HM, et al. Survival outcome of weak estrogen/progesterone receptor expression in HER2 negative breast cancer is similar to triple negative breast cancer. Eur J Surg Oncol. 2017; 43(10): 1855-1861.

7. Inwald EC, Koller M, Klinkhammer-Schalke M, et al. 4IHC classification of breast cancer subtypes in a large cohort of a clinical cancer registry: use in clinical routine for therapeutic decisions and its effect on survival. Breast Cancer Res Treat. 2015; 153(3): 647658.

8. Kremani TA, Kermani IA, Faham Z, Dolatkhah R. Ki-67 status in patients with primary breast cancer and its relationship with other prognostic factors. Biomed Res Ther. 2019; 6(2): 2986 - 2991.

9. Mirmalek SA, Ghorbani M, Boushehrinejad AG, Salehi M, Salimi-Tabatabaee SA, Aryan H. Correlation of Ki67 expression with hormone receptors, human epidermal growth factor receptor-2 (HER-2) status, P53 mutation and clinicopathological characteristics in pathological specimens of breast cancer patients. Galen Med J. 2016; 5(2): 90-97.

10. Sheikhpour R, Poorhosseini F. Relation between estrogen and progesterone receptor status with P53, Ki-67 and HER-2 markers in patients with breast cancer. Ir J Blood Cancer. 2016; 8(4): 93-97.

11. Hugh J, Hanson J, Cheang MC, et al. Breast cancer subtypes and response to docetaxel in node-positive breast cancer: use of an immunohistochemical definition in the BCIRG 001 trial. J Clin Oncol. 2009; 27(8): 1168-1176.

12. Chung YR, Jang MH, Park SY, Gong G, Jung WH; Korean Breast Pathology Ki-67 Study Group. Interobserver variability of Ki-67 measurement in breast cancer. J Pathol Transl Med. 2016; 50(2): 129137.

13. Thangarajah F, Enninga I, Malter W, et al. A retrospective analysis of Ki-67 index and its 
prognostic significance in over 800 primary breast cancer cases. Anticancer Res. 2017; 37(4): 1957-1964.

14. Yang J, Long Q, Li H, Lv Q, Tan Q, Yang X. The value of positive lymph nodes ratio combined with negative lymph node count in prediction of breast cancer survival. J Thorac Dis. 2017; 9(6): 1531 - 1537.
15. Denkert C, Loibl S, Muller BM, et al. Ki-67 levels as predictive and prognostic parameters in pretherapeutic breast cancer core biopsies: a translational investigation in the neoadjuvant GerparTrio trial. Ann Oncol. 2013; 24(11): 2786-2793. 